\title{
The effect of education on improving hand hygiene compliance among healthcare workers
}

\begin{abstract}
Background: Proper hand hygiene is the most effective way to reduce healthcareassociated infections (HAI). Hand hygiene compliance rates among healthcare workers remain unsatisfactory. This study investigated the effects of unit in-service education on hand hygiene compliance in an Interventional Radiology and Cardiac Catheterization Laboratory department in a tertiary hospital in Florida.
\end{abstract}

Methods: An observational study was conducted for five weeks. A total of 120 participants, consisting of 40 physicians, 40 registered nurses, 40 technologists were observed by a secret shopper pre-education and post education. A pre-education baseline compliance rate was established for each group. Pre-education observation occurred for two weeks before the intervention, one week for the implementation of unit in-service education, another two weeks for post education observation. The hand hygiene audit tool was utilized to record the observation data.

Data analysis: Quantitative data were analyzed using a one-tailed T-test to determine P values for each group.

Results: Results showed a $20 \%$ increase among physicians, $15 \%$ among nurses and 5\% among technologists. There was a statistical significance for the physician and nurse groups.

Conclusion: The study implies that education is an effective intervention in improving hand hygiene compliance rates among the staff in the interventional radiology and cardiac catheterization department.

Keywords: hand hygiene compliance, hand hygiene, education, healthcare workers, healthcare-associated infections
Volume 3 Issue 2 - 2019

\author{
Mary Thisbe Quilab, Shamarra Johnson, \\ Christian Schadt, Abigail Mitchell \\ Simmons University, USA
}

Correspondence: Abigail Mitchell RN, Simmons University, USA, Tel 7|6-829-82|8, Email mitchela@dyc.edu

Received: March 02, 2019 | Published: April 02, 2019

\section{Introduction}

The rate of healthcare-associated infections has been on the rise resulting in prolonged hospital stay and economic burden to the healthcare system. Health-associated infections (HAI) are a significant cause of increased morbidity and mortality. Substandard hand hygiene practices increase the risk of health-associated infections. One of the best practices to prevent healthcare-associated infections is hand hygiene technique. Studies have shown that the hand hygiene compliance rate among healthcare workers has been disturbingly low, leading to an increase in healthcare-associated infections. The research question for this quality improvement project: Does providing quarterly hand hygiene education to the staff working in the Interventional Radiology and Cardiac catheterization laboratory department improve hand hygiene compliance rates? The World Health Organization (WHO) estimated that hand hygiene compliance among healthcare workers is between $5 \%$ and $81 \%$, with $40 \%$ as the average. Almost two million healthcare-associated infections occur every year across the United States, according to the Centers for Control and Disease Prevention. Hand hygiene is recognized to be the single factor in reducing healthcare-associated infections (HAIs). According to the Centers for Control and Disease and Prevention, ${ }^{1}$ roughly, healthcare workers do not wash their hand as often as possible, which contributes to the increasing number of healthcare-associated infections. The CDC also reported that 1 in 25 hospital patients develop health-associated infections in any given day. Globally, millions of patients are affected by health-associated infections yearly. ${ }^{2}$ Infections result in prolonged hospital stay, severe illness, high hospital costs and induce long-term disabilities which could contribute to a massive and financial burden to the patients, families and the healthcare system. Compliance with safe hand hygiene practices is unsatisfactorily low among healthcare workers worldwide. Currently, the baseline compliance rate of hand hygiene in the Interventional Radiology and Cardiac Catheterization Laboratory department is only $79 \%$. The standard hand hygiene compliance should be above $91 \%$, as recommended by the World Health Organization. Hence, the objective of this quality improvement project was to evaluate the effect of a quarterly unit inservice education on improving hand hygiene compliance rate among the staff in the Interventional Radiology and Cardiac Catheterization Laboratory department in a tertiary hospital in Florida.

\section{Nursing theorist}

Florence Nightingale, the first nursing researcher, is the founder of modern nursing. She was an English, social reformer who was born in Florence, Italy. Nightingale organized the care of the wounded soldiers during the Crimean war in the p1800's. She was making rounds and checking the wounded soldiers at night; hence she gained the title "The Lady with the Lamp." Florence Nightingale established the first nursing school in the world in the year 1860 which then became the foundation of professional nursing. ${ }^{3}$ Some of Nightingale's outstanding works were sanitary reform and visual presentation of statistics. She created the first nurses' notes that eventually became the basis of scientific nursing research and practice. Her lasting contribution to modern nursing sets an example of compassion and commitment to patient care. Florence Nightingale developed the 
Environmental Theory in the second half of the nineteenth century; the theory focuses on the environmental factors that affect the health of patients. Florence Nightingale proposed that the environment has a strong influence on patient outcomes. Therefore, poor hand hygiene can pose a direct threat to patient outcomes by placing them at risk for healthcare-associated infections.

\section{Literature review}

A literature search process was performed from nursing databases on hand hygiene compliance improvement strategies The primary databases used for the search were: Cochrane Systematic Reviews, Cumulative Index to Nursing \& Allied Health Literature (CINAHL) MEDLINE, PubMed, Embase, and Google Scholar. The key search terms used were hand hygiene compliance, healthcare workers, hand hygiene, knowledge, hand hygiene practices, healthcare-associated infections. Research studies published within the last five years, from the year 2013-2018 were selected.

\section{Hand hygiene}

Hand hygiene aims to combat the spread of infection. Nosocomial infections are a burden on our health care system. These kinds of infections increase the length of hospital stay, cause harm, and can impact the financial situation on our health care system significantly. ${ }^{4}$ This meta-analysis demonstrated by the researchers showed that $\$ 9.8$ billion annually was spent by hospitals in the United States due to the five leading nosocomial infections (healthcare-associated infections, catheter-associated urinary tract infections, ventilator-associated pneumonia, surgical site infections, Clostridium Difficile, and central line-associated bloodstream infections). Nosocomial infections are one of the most preventable sources of harm, more strategies to prevent these infections should be the focus. A prospective cohort looked at the cost and length of stay associated with nosocomial infection. It was determined that these infections doubled the cost of the hospital stay and doubled the length of stay versus those without a nosocomial infection. ${ }^{5}$

A study by Fox et al. ${ }^{6}$ indicated that a hand hygiene protocol was useful in reducing healthcare-associated infections (HAIs). The study adopted a pre-experimental study design where a patient hand hygiene protocol was the intervention whose effectiveness was evaluated after 12 months. The research, however, did not focus on education as a strategy that could ensure hand hygiene compliance and associated significant positive effects. Even though a form of education was utilized in this study, it adopted a punitive approach instead of a practical approach. It is easier to implement change when individuals realize the benefit of the change as opposed to forcing individuals to do something. Niyonzima et al. ${ }^{7}$ showed that educational intervention only is not enough to achieve the intended change. Understanding the challenges and barriers that might impede the proposed amendment is equally important to minimize resistance and encourage adoption to the transition.

\section{Barriers}

Preceding interventions related to increasing hand hygiene compliance, barriers to compliance were considered. Healthcare workers are not deliberately spreading infections through ineffective hand washing and may require tools or resources to make the process more efficient. Molinari ${ }^{8}$ identified several factors that lead to hand hygiene noncompliance among healthcare workers. His list included understaffing, lack of soap or paper towels, insufficient time, and perceived the low risk of cross-infection. One of the most influential factors that influence hand hygiene noncompliance is skin irritation and dermatitis. The pain and irritation from hand washing in abrasive cleansers several times during a shift prevent healthcare workers from effectively washing their hands before, during, and after patient care. In response, many facilities are incorporating gentle cleansers, waterless alcohol-based rubs, and hand lotions address the issue of dermatitis. ${ }^{8}$ Another barrier to hand hygiene in healthcare organizations mentioned by Molinari ${ }^{8}$ was the lack of education and knowledge regarding hand hygiene principles, guidelines, and protocols. Evidence supporting this observation has led many organizations to increase education and awareness of effective hand hygiene among healthcare workers.

An article by Andersson et al. ${ }^{9}$ also illustrates the importance of addressing barriers and challenges to achieve success for a change initiative. It is typical for staff members to resist change, but when the right approach is used, beneficial outcomes are realized in facilitating the move. Unlike in the study by Fox et al. ${ }^{6}$, Andersson et al. ${ }^{9}$ lay the foundation for subsequent research that aims to use education as a way of improving hand hygiene compliance among health workers. Andersson et al. ${ }^{9}$ indicate that individuals should be allowed to express their opinions because when these opinions are taken into considerations and the fears of the targeted people addressed, the result is motivated and enthusiastic people. Once again, Pan et al. ${ }^{10}$ indicated the essence of using an educational approach that motivates healthcare workers to increase compliance with hand hygiene protocols of the facilities in which they work. The intensity of an educational program is not as effective as supporting and providing feedback to the targeted healthcare workers.

\section{Improving compliance rates}

Reduced hand hygiene compliance among healthcare professionals is considered a global problem, even though hand hygiene is very plain and straightforward. ${ }^{11}$ The study conducted by Nteli et al. ${ }^{11}$ among healthcare workers in a pediatric intensive care unit demonstrated an increase of $\mathrm{HH}$ compliance $31.8 \%$ to a staggering $67.7 \%$. Similar studies have shown increased compliance after an educational intervention. Although the compliance rate was increased instantly after the first educational intervention, an essential finding of this study is that after six months after the first educational intervention, the compliance rate has declined. Hand hygiene compliance differs considerably among healthcare professions. Many studies found out that nurses have increased compliance than doctors. The research of Nteli et al. ${ }^{11}$ agreed that doctors have a low compliance rate than nurses. It is challenging for most healthcare facilities to improve hand hygiene compliance of staff. Hand hygiene compliance is a state of mind and behavioral.

According to Midturi et al. ${ }^{12}$ increasing awareness of hand hygiene at any level contributes to improved compliance among healthcare workers. They found that a multimodal approach led by the administrative leadership resulted in the increased sustainability of hand hygiene compliance. Based on these findings, Midturi et al. ${ }^{12}$ suggested to encourage the involvement of administrative leadership in creating hand hygiene compliance strategies and healthcare facilities should develop an individualized approach to promote sustainability of hand hygiene compliance. Another study by Al-Khawaldeh et al. ${ }^{13}$ found that knowledge, beliefs, and attitudes are significant predictors of hand hygiene compliance. Moreover, hand hygiene education courses are needed to improve the competency of nursing students in dealing with infection control issues. ${ }^{12}$

Several approaches to increasing hand hygiene compliance among healthcare workers have been implemented thus far. Some 
of those interventions, as demonstrated by Midturi et al. ${ }^{12}$ include education, direct monitoring, visual queuing, consistent feedback, staff recognition, and effective management. According to the World Health Organization ${ }^{2}$ hand hygiene compliance is a global initiative prioritized by healthcare organizations worldwide. Therefore, several healthcare facilities have researched and employed several interventions to address hand hygiene noncompliance in conjunction with resulting HAIs. While some approaches to improving hand hygiene are multifaceted, some focus on a single intervention. By employing a quantitative research design, Daisy \& Sreedevi ${ }^{14}$ were able to demonstrate improved hand washing compliance rates by implementing a multifocal educational strategy administered. Results demonstrated good knowledge among all the nurses and a significant improvement in compliance rates after the educational intervention.

Another way of educating healthcare professionals on hand hygiene $(\mathrm{HH})$ to improve compliance is the utilization of visual aids such as posters. Akingbola et al. ${ }^{15}$ determined the impact of hand hygiene posters on compliance on resident physicians' compliance in the pediatric intensive care unit. The overall compliance rate in this study improved to $44 / 7 \%$ from $22.22 \%$ baseline, which was still significantly low. It was found that the hand hygiene compliance rate increased among pediatric residents compared to the surgical residents after the introduction of $\mathrm{HH}$ posters. Additional patient care responsibilities and time pressure of surgical residents played a role in the decreased compliance rate. ${ }^{15}$ Furthermore, Akingbola et al. ${ }^{15}$ argued that diminished educational influence or the manifestation of Hawthorne effect could be the contributing factors of lack of apparent change of $\mathrm{HH}$ compliance rate between interventions.

\section{Summary}

Hand hygiene aims to combat the spread of infection. Nosocomial infections are a burden on our health care system. These kinds of infections increase the length of hospital stay, cause harm, and can impact the financial situation on our health care system significantly. ${ }^{4}$ This meta-analysis demonstrated by the researchers showed that $\$ 9.8$ billion annually was spent by hospitals in the United States due to the five leading nosocomial infections (healthcare-associated infections, catheter-associated urinary tract infections, ventilator-associated pneumonia, surgical site infections, Clostridium Difficile, and central line-associated bloodstream infections). Nosocomial infections are one of the most preventable sources of harm, more strategies to prevent these infections should be the focus. A prospective cohort looked at the cost and length of stay associated with nosocomial infection. It was determined that these infections doubled the cost of the hospital stay and doubled the length of stay versus those without a nosocomial infection. ${ }^{5}$

\section{Methods}

A quantitative observational study design was used to determine hand washing compliance rates pre and post educational intervention. The study took place in the interventional radiology and cardiac catheterization lab of a tertiary hospital in Florida. No IRB required, as this quality improvement study was approved by the unit manager. The participants were all the staff working in the Catheterization Laboratory and Interventional Radiology unit which comprised of physicians, registered nurses, and radiology technicians. The overall compliance of the participants was observed two weeks before the implementation of educational in-service to establish the current hand hygiene compliance rates as a baseline during routine patient care. The educational intervention, consisting of the Joint Commission on Accreditation of Healthcare Organizations (JCAHO) and the World
Health Organization (WHO) guidelines, was implemented throughout one week. Participants were observed again for two weeks directly following educational intervention. Participants were discreetly monitored pre and post education by the researcher and delegated staff member called the secret shopper. A hand washing rating tool was used consistently throughout the study to record the compliance results (Figure 1-4). The researcher was also careful to ensure that an equal number of the three groups, physicians, nurses, and technicians during the pre and post educational intervention period were observed. ${ }^{16-20}$

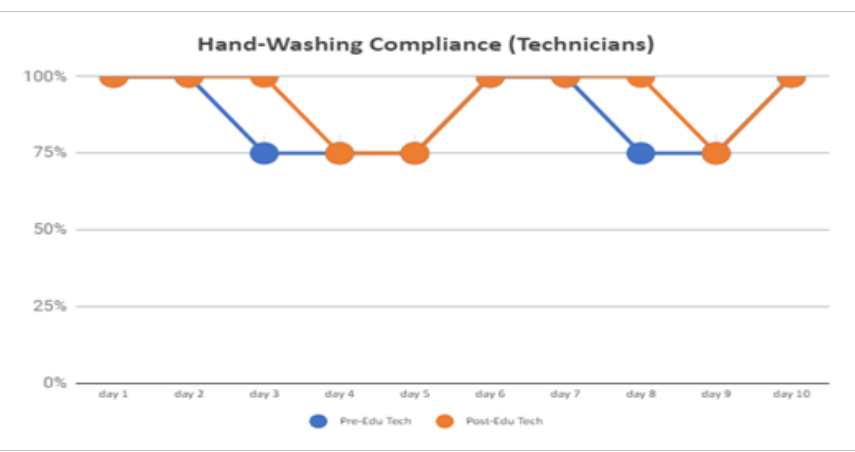

Figure I Hand washing compliance pre and post education for technicians.

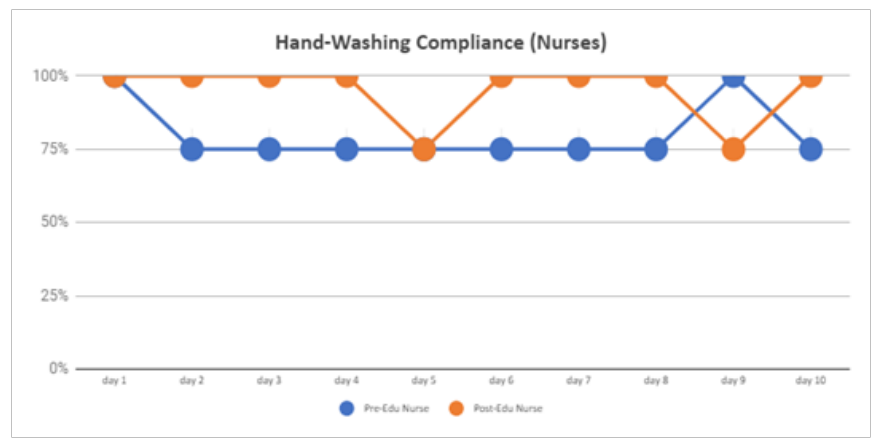

Figure 2 Hand washing compliance pre and post education for nurses.

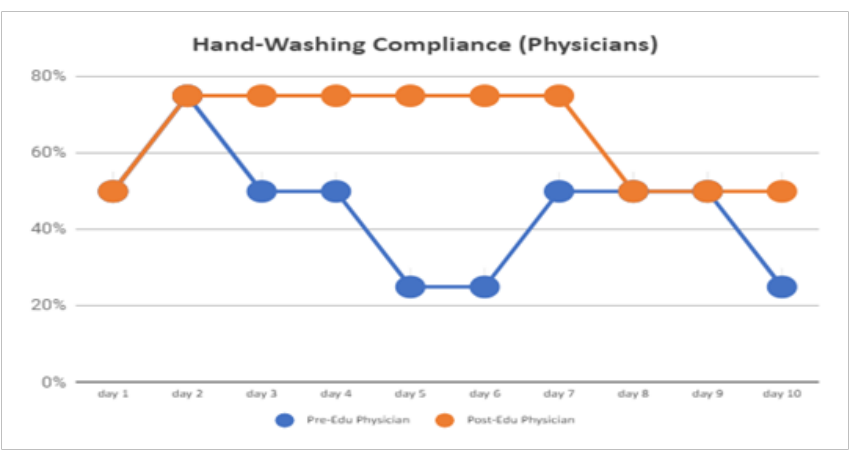

Figure 3 Hand washing compliance pre and post education for physicians.

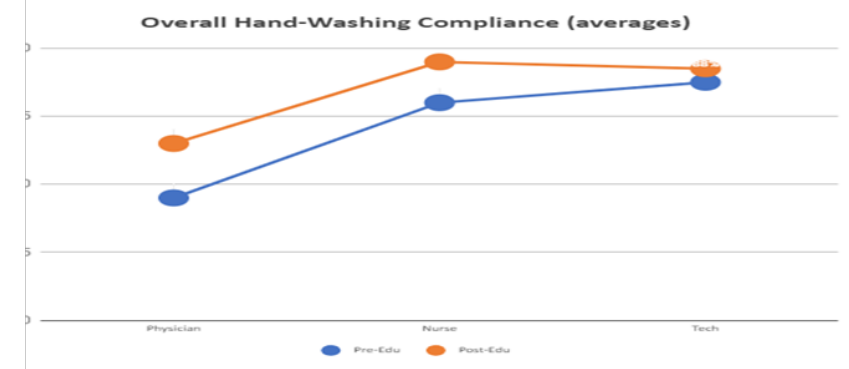

Figure 4 Average hand washing compliance pre and post education for all staff. 


\section{Data analysis}

Data pre and post educational intervention were analyzed for each group using a one-tailed T-test. The t-test was done using daily averages for each group. A standard $\mathrm{P}$ value of 0.05 was used as a benchmark for the resulting $\mathrm{P}$ values of each group to determine statistical significance. P values less than 0.05 were deemed statistically significant whereas $\mathrm{P}$ values greater than 0.05 were determined to have no statistical relevance. The data were also arranged in graphs and scatter plots in order to show a visual comparison amongst the groups (Graph 1 \& 2).

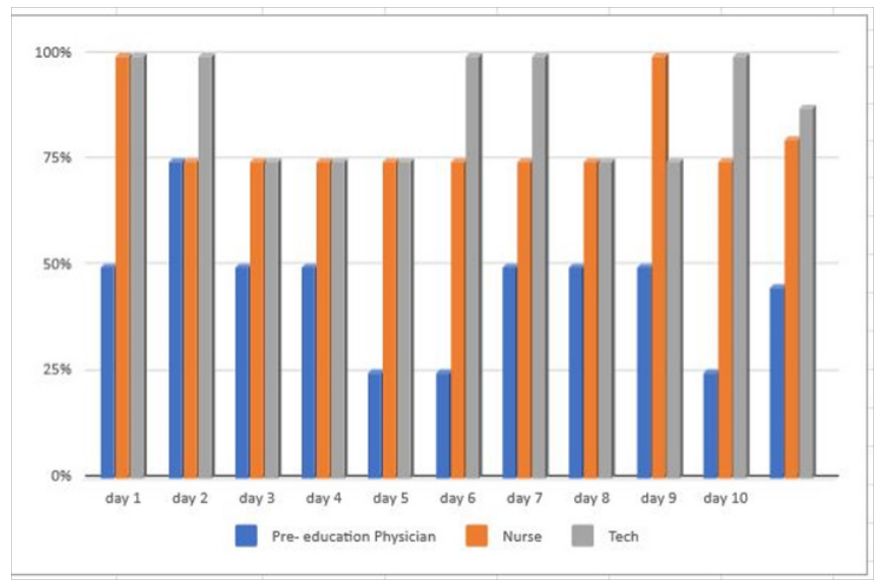

Graph I Pre education compliance rates.

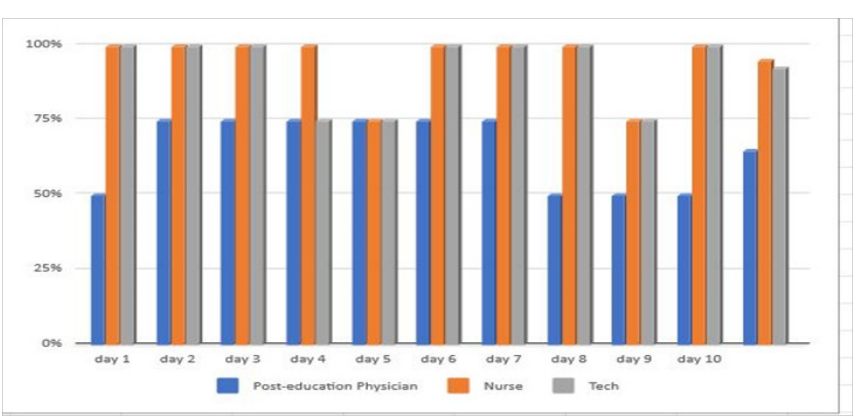

Graph 2 Post education compliance rates.

\section{Results}

Initial analysis of the data showed an increase in hand hygiene compliance rates among all groups observed in the study. Closer analysis revealed an average increase of 20 percent among physicians, 15 percent among nurses, and 5 percent among technician (Table 1). $\mathrm{P}$ values were calculated using a one-tailed T-test between groups assuming equal variance. The results revealed a $\mathrm{P}$ value of 0.01070801996 for physicians (Table 2), a P value of 0.02385638454 for nurses (Table 3), and a P value 0.1678506561 for technicians (Table 4) when comparing hand washing compliance rates for these groups pre and post educational intervention. A P value of 0.230863652 was calculated when comparing averages between the three groups. The standard $\mathrm{P}$ value of less than .05 was applied to the $\mathrm{P}$ values of each group to determine statistical significance. According to the $\mathrm{P}$ values, there is a statistically significant difference in hand hygiene compliance rates post staff education for the physician and nurse groups in the experiment. However, the study was unable to show a statistical significance for the technician group post educational intervention.
Table I Average compliance rates

\begin{tabular}{llll}
\hline $\begin{array}{l}\text { Average } \\
\text { compliance rate }\end{array}$ & Pre-Edu & Post-Edu & $\begin{array}{l}\text { Compliance } \\
\text { rate difference }\end{array}$ \\
\hline Physician & $45 \%$ & $65 \%$ & $20 \%$ \\
Nurse & $80 \%$ & $95 \%$ & $15 \%$ \\
Tech & $88 \%$ & $93 \%$ & $5 \%$ \\
\hline
\end{tabular}

Table 2 T-test physicians

\begin{tabular}{llll}
\hline & $\begin{array}{l}\text { Pre-Edu } \\
\text { physician }\end{array}$ & $\begin{array}{l}\text { Post-Edu } \\
\text { physician }\end{array}$ & $\begin{array}{l}\text { Compliance rate } \\
\text { difference }\end{array}$ \\
\hline Day I & $50 \%$ & $50 \%$ & $0 \%$ \\
Day 2 & $75 \%$ & $75 \%$ & $0 \%$ \\
Day 3 & $50 \%$ & $75 \%$ & $25 \%$ \\
Day 4 & $50 \%$ & $75 \%$ & $25 \%$ \\
Day 5 & $25 \%$ & $75 \%$ & $50 \%$ \\
Day 6 & $25 \%$ & $75 \%$ & $50 \%$ \\
Day 7 & $50 \%$ & $75 \%$ & $25 \%$ \\
Day 8 & $50 \%$ & $50 \%$ & $0 \%$ \\
Day 9 & $50 \%$ & $50 \%$ & $0 \%$ \\
Day 10 & $25 \%$ & $50 \%$ & $25 \%$ \\
Mean & & & $20 \%$ \\
Standard deviation & & 0.1870828693 \\
P-value & & & 0.01070801996 \\
Significance level & & $<0.05$ \\
\hline
\end{tabular}

Table 3 T-test nurses

\begin{tabular}{llll}
\hline & $\begin{array}{l}\text { Pre-Edu } \\
\text { nurse }\end{array}$ & $\begin{array}{l}\text { Post-Edu } \\
\text { nurse }\end{array}$ & $\begin{array}{l}\text { Compliance rate } \\
\text { difference }\end{array}$ \\
\hline Day I & $100 \%$ & $100 \%$ & $0 \%$ \\
Day 2 & $75 \%$ & $100 \%$ & $25 \%$ \\
Day 3 & $75 \%$ & $100 \%$ & $25 \%$ \\
Day 4 & $75 \%$ & $100 \%$ & $25 \%$ \\
Day 5 & $75 \%$ & $75 \%$ & $0 \%$ \\
Day 6 & $75 \%$ & $100 \%$ & $25 \%$ \\
Day 7 & $75 \%$ & $100 \%$ & $25 \%$ \\
Day 8 & $75 \%$ & $100 \%$ & $25 \%$ \\
Day 9 & $100 \%$ & $75 \%$ & $25 \%$ \\
Day 10 & $75 \%$ & $100 \%$ & $25 \%$ \\
Mean & & & $15 \%$ \\
Standard deviation & & & 0.1658312395 \\
P-value & & & 0.02385638454 \\
Significance level & & & $<0.05$ \\
\hline
\end{tabular}

If null hypothesis is $p \geq 0.05$, then we failed to reject the null hypothesis

There is no significant before and after education. 
Table 4 T-test technicians

\begin{tabular}{llll}
\hline & $\begin{array}{l}\text { Pre-Edu } \\
\text { tech }\end{array}$ & $\begin{array}{l}\text { Post-Edu } \\
\text { tech }\end{array}$ & $\begin{array}{l}\text { Compliance rate } \\
\text { difference }\end{array}$ \\
\hline Day I & $100 \%$ & $100 \%$ & $0 \%$ \\
Day 2 & $100 \%$ & $100 \%$ & $0 \%$ \\
Day 3 & $75 \%$ & $100 \%$ & $25 \%$ \\
Day 4 & $75 \%$ & $75 \%$ & $0 \%$ \\
Day 5 & $75 \%$ & $75 \%$ & $0 \%$ \\
Day 6 & $100 \%$ & $100 \%$ & $0 \%$ \\
Day 7 & $100 \%$ & $100 \%$ & $0 \%$ \\
Day 8 & $75 \%$ & $100 \%$ & $25 \%$ \\
Day 9 & $75 \%$ & $75 \%$ & $0 \%$ \\
Day 10 & $100 \%$ & $100 \%$ & $0 \%$ \\
Mean & & & $5 \%$ \\
Standard deviation & & $0.1 \%$ \\
P-value & & & 0.1678506561 \\
Significance level & & $>0.05$ \\
\hline
\end{tabular}

If null hypothesis is $p \geq 0.05$, then we failed to reject the null hypothesis

There is no significant.

\section{Limitations}

A few limitations where identified in this study. The time allocated for the study was not long enough to obtain optimal compliance results, only a total of six weeks to conduct the study. The sample size was small, consisting of 120 participants, and the study was limited to the Catheterization Lab and Interventional Radiology department. FNP Nursing student allotted two weeks to provide education to the staff; however, the education in-service was presented only within a week due to the time of the study implementation which was conducted during a holiday season. Some staff was unavailable to attend the educational in-service. Another limitation is the education portion of the study was a verbal presentation only without any posters left for visual cues.

\section{Implications to nurse practitioner practice}

There are several strategies the nurse practitioner can employ to contribute to HAI prevention and proper hand hygiene practices among the healthcare team. Using Florence Nightingale's environmental theory, the nurse practitioner is aware of what constitutes the patient environment and understands how interactions within it contribute to possible infection. Besides, the knowledge gained from Nightingale's theory provides a basis for HAI prevention. As a result, the nurse practitioner maintains proper hand hygiene practices and helps colleagues remain accountable to the same standards when applicable. In the event a hospital-acquired infection does occur, the nurse practitioner is well-equipped with the skills and authority necessary to effectively treat the illness. The relationship between the patient and provider is paramount in establishing trust between the two, providing ease of communication which allows the nurse practitioner to empower patients with knowledge regarding good hand hygiene. Patients are then able to contribute to their healthcare management by becoming active participants in preventing HAIs through the promotion of proper hand hygiene practices for themselves and others in their environment. Furthermore, nurse practitioners can play a crucial role in improving hand hygiene practices by implementing hand hygiene education to other medical professionals and to the patients they serve.

\section{Conclusion}

In summary, the in-service educational intervention produced an improvement of hand hygiene compliance among the staff in the Catheterization Laboratory and Interventional Radiology unit. We concluded that hand hygiene educational in-service was an effective method in increasing hand hygiene compliance among all personnel. The participants which included physician registered nurses and technologists demonstrated increased rates of hand hygiene compliance post educational intervention. There are different methods for improving hand hygiene compliance. More research is needed to ascertain the most effective methods to increase hand hygiene compliance, therefore minimizing healthcare-associated infections. The use of posters and visual aids emphasizing the importance of hand hygiene should be posted in every unit. We further recommend that educational in-services should be implemented regularly in the healthcare facilities and in every unit to maintain the sustainability of compliance rates. Every unit needs to delegate a group of staff to monitor hand hygiene compliance rates and to provide educational intervention regularly. More research with larger sample size checking $\mathrm{HHC}$ rates at intervals post interventions to determine the longevity of education. Another excellent study would to compare different education models such as verbal presentations, written instructions, posters and video presentations (verbal, written, poster, etc. and determine which increases hand hygiene compliance greater. Hand hygiene is a very enthralling and controversial issue that needs more inquiry. Research to evaluate long term and sustainable solutions to increase hand hygiene compliance is a needed area to explore.

\section{Acknowledgments}

None.

\section{Conflicts of interest}

The authors declare there is no conflict of interest.

\section{References}

1. Centers for Disease and Control Prevention. 2016.

2. World Health Organization. The Evidence for Clean Hands. Infectious Disease and Control. 2018.

3. Florence Nightingale-Nursing Theorist. 2016.

4. Zimlichman E1, Henderson D, Tamir O, et al. Health care-associated infections a meta-analysis of costs and financial impact on the US health care system. JAMA Intern Med. 2013;173(22):2039-2046.

5. Chacko B, Thomas K, David T, et al. Attributable cost of a nosocomial infection in the intensive care unit: A prospective cohort study. World $J$ Crit Care Med. 2017;6(1):79-84.

6. Fox C, Wavra T, Drake DA, et al. Use of a patient hand hygiene protocol to reduce hospital-acquired infections and improve nurses' hand washing. Am J Crit Care. 2015;24(3):217-223.

7. Niyonzima V, Beinempaka F. Practice and compliance of essential handwashing among healthcare workers at a regional referral hospital in Uganda: A quality improvement and evidence-based practice. Canadian Journal of Infection Control. 2018;33(1):33-38. 
8. Molinari JA. Effective, sensible, and safe hand hygiene: Poor compliance among clinicians still leads to associated infections. $R D H$. 2017;37(10):62-65.

9. Andersson A, Frodin M, Dellenborg L, et al. Iterative co-creation for improved hand hygiene and aseptic techniques in the operating room: Experiences from the safe hands study. BMC Health Serv Res. 2018;18(2):1-12.

10. Pan SC, Tien KL, Hung IC, et al. Compliance of health care workers with hand Hygiene practices: Independent advantages of overt and covert observers. PLoS One. 2013;8(1):e53746.

11. Nteli C, Galanis P, Koumpagioti D, et al. Assessing the effectiveness of an educational program on compliance with hand Hygiene in a pediatric intensive care unit. Advances in Nursing. 2014:1-4.

12. Midturi JK, Narasimhan A, Barnett T, et al. A successful multifaceted strategy to improve hand hygiene compliance rates. Am J Infect Control.2015;43(5):533-536.

13. Al-Khawaldeh OK, Al-Hussami M, Darawad M. Influence of nursing students handwashing knowledge, beliefs, and attitudes on their handwashing compliance. Health. 2015;7(5):572-579.

14. Daisy VT, Sreedevi TR. Effectiveness of a multi-component educational intervention on knowledge and compliance with hand hygiene among nurses in neonatal intensive care units. International Journal of Nursing Education. 2015;7(4):98-103.
15. Akingbola O, Singh D, Srivastav S, et al. The impact of hand hygiene posters on hand hygiene compliance rate among resident physicians: A brief report. Clinical Pediatrics. 2016;1(4):1-2.

16. Doronina O, Jones D, Martello M, et al. A systematic review on the effectiveness of interventions to improve hand hygiene compliance of nurses in the hospital setting. J Nurs Scholarsh. 2017;49(2):143-152.

17. Graveto JM, Rebola R, Fernandes E, et al. Hand hygiene: nurses' adherence after training. Rev Bras Enferm. 2018;71(3):1189-1193.

18. Koo E, McNamara S, Lansing B, et al. Making infection prevention education interactive can enhance knowledge and improve outcomes: Results from the Targeted Infection Prevention (TIP) Study. Am J Infect Control. 2016;44(11):1241-1246.

19. Phan HT, Tran HTT, Tran HTM, et al. An educational intervention to improve hand hygiene compliance in Vietnam. BMC Infect Dis. 2018;18(1):116.

20. Sahai V, Eden K, Gluestein S. Hand hygiene knowledge, attitudes and self-reported behaviour in family medicine residents. Canadian Journal of Infection Control. 2016;31(1):11-17. 